\section{G. Deegener}

Aggression und Gewalt von Kindern und Jugendlichen.

Ein Ratgeber für Eltern, Lehrer und Erzieher

Göttingen, Hogrefe, 2002, 132 S., EUR 15,95

ISBN 3-8017-1607-4

Aggression und Gewalt, kaum ein anderes Thema ist so gegenwärtig und beschäftigt uns so nachhaltig wie dieses. Tagtäglich werden wir in den Zeitungen, im Radio oder Fernsehen damit konfrontiert - und zunehmend, so scheint es, sind es Jugendliche und Kinder, die in diesem Zusammenhang in Erscheinung treten. Jedoch nicht nur als Opfer, sondern auch als Täter. Der Name der Stadt Erfurt steht dafür beispielhaft sicher als eines der schrecklichsten Ereignisse in jüngster Zeit. Aber auch in unserem nächsten Umfeld, in den Familien, in der Nachbarschaft, auf der Straße, in den Schulen oder auch Kindergärten begegnet man immer wieder Aggressionen verschiedenster Form und verschiedensten Ausmaßes. Wie kommt es zu Aggressionen und Gewalt? Welche Ursachen und Erklärungsmodelle finden sich und was können wir dagegen tun? Gibt es wirklich immer mehr Gewalt auch schon unter Kindern und Jugendlichen? Genau diese Fragen stellt Günther Deegener in seinem Buch und versucht, dieses Thema in 12 Kapiteln von möglichst vielen Seiten zu beleuchten. Das Buch ist relativ übersichtlich gestaltet und lässt sich gut und leicht, dem Anspruch eines Ratgebers folgend, lesen. Nach einer kurzen Einleitung, die neben einem Überblick über die Inhalte des Buches dazu ermuntert, Gewalt von Kindern und Jugendlichen differenziert zu betrachten, folgen im 2. Kapitel neben allgemein- und rechtsgültigen Definitionen von Gewalt bzw. Gewaltanwendung sowie Aggression erste Überlegungen zu Ursachen und Motiven. Dabei führt der Autor neben Fallbeispielen und Zitaten auch Ergebnisse von Studien auf. Auch in den folgenden Kapiteln werden immer wieder Beispiele angeführt und die Aussagen des Autors durch Studien und Zitate belegt oder ergänzt.

Kapitel 3 beschäftigt sich mit der Häufigkeit von Gewalt bei Kindern und Jugendlichen, wobei zum einen nach Schweregrad und Ausprägung unterschieden und zum anderen zum Nachdenken angeregt wird, einen Mittelweg zwischen «Verharmlosung und Dramatisierung» von Kinder- und Jugendgewalt zu finden. Die folgenden drei Kapitel zeigen Ursachen und Folgen auf und stellen Zusammenhänge zwischen Gewalt im Elternhaus und selbst verübten Gewalttaten her. Dabei wird in den Kapiteln 4 und 5 der Schwerpunkt auf sexuelle Gewalt und Aggression gelegt und im 6. Kapitel mögliche ver- bale, seelische und körperliche Gewalt im Erziehungsalltag der Eltern dargestellt. Das Spannungsfeld zwischen einem sehr gewährenden und wenig eingrenzenden Erziehungsstil einerseits und zu engen und strafenden Erziehungsmethoden andererseits sowie deren mögliche direkte und indirekte Folgen wird aufgezeigt. Auch im 7. Kapitel geht es um Gewalt und Aggression im Erziehungsalltag, wobei die natürlicherund notwendigerweise vorkommenden Phasen von Wut, Aggression und Trotz vor allem im jüngeren Kindesalter als wichtige Bausteine für eine normale Entwicklung herausgestellt werden. Um Jugendarrest und geschlossene Heime sowie Alternativen dazu geht es in den Kapiteln 8 und 9 vor dem Hintergrund gesamtgesellschaftlicher Überlegungen und notwendiger infrastruktureller Veränderungen, um Gewalt primär vorbeugen zu können. In den letzten drei Kapiteln schließlich gibt der Autor praktische Alltagstipps für Eltern und Lehrer verbunden mit konkreten Handlungsanweisungen und Beispielen von kontinuierlicher «Elternbildung» in Form von so genannten «Elternbriefen». Dabei werden diese Elternbriefe nicht nur ausführlich beschrieben, sondern auch Bezugsquellen benannt.

Einziger Kritikpunkt sind die teilweise unvollständigen oder fehlenden Quellenangaben der zitierten Statistiken, Studien oder Befragungen. Insgesamt bietet dieser Ratgeber jedoch einen guten Überblick zum Thema Gewalt und Aggression von Kindern und Jugendlichen, der durchaus nicht nur für die angesprochenen Gruppen lesenswert ist und in einem guten Preis-Leistungs-Verhältnis steht.

Sandra Gerstner, Mannheim

A. Franke; A. Kämmerer (Hrsg.)

\section{Klinische Psychologie der Frau. Mit einer Einführung von Lilly Kemmler}

Göttingen, Hogrefe, 2001, 778 S., EUR 49,95

ISBN 3-8017-1333-4

Gibt es eine Psychologie der Frau? Selbstverständlich! Aber gibt es auch eine klinische Psychologie der Frau? Diese Frage versuchen 27 Autorinnen (darunter übrigens 5 Männer) zu beantworten.

Zunächst zum Inhalt: Nach einem einführenden Kapitel zu Gesundheits- und Krankheitstheorien der klinischen Psychologie und ihrer Anwendung auf Frauen sowie allgemeinen Überlegungen zu Epidemiologie, Diagnostik und Ätiologie

\section{KARGER}

Fax +497614520714

E-mail Information@Karger.d

www.karger.com

(c) 2003 S. Karger GmbH, Freiburg 
psychischer Störungen bei Frauen folgt der Hauptteil, in dem die einzelnen Störungsbilder orientiert an unseren gängigen Diagnoseschemata dargestellt werden. Es folgt ein Kapitel zu Ressourcen, Belastungen und Vulnerabilitäten im weiblichen Lebensverlauf und ein abschließendes Kapitel zu Aspekten der psychosozialen Versorgung.

Wir haben dieses Buch als Ehepaar, das im Rahmen der psychotherapeutischen Versorgung tätig ist, gelesen und diskutiert. Nach der Lektüre steht für uns fest: Es gibt eine klinische Psychologie der Frau, ja, es muss eine klinische Psychologie der Frau geben. Zu unterschiedlich in Ausprägung und Häufigkeit sind psychische Störungen bei den beiden Geschlechtern vertreten, zu unterschiedlich ist die Bereitschaft und die Fähigkeit, Probleme als psychisch bedingt zu akzeptieren und zu reflektieren und damit das so genannte Inanspruchnahmeverhalten.

Noch ein zweiter Aspekt wurde uns bei der Lektüre bewusst: Die «klinische Psychologie» ist traditionell männlich dominiert: Mensch gleich Mann. Was Gesundheit ist, wird nach männlichen Maßstäben definiert. Männliche Rollencharakteristika werden gesellschaftlich höher bewertet und gelten als gesünder, während weibliche Verhaltensweisen als neurotisch diskriminiert werden. Weibliche Sichtweisen eröffnen hier Perspektiven, die Männern anscheinend verschlossen, nicht bewusst sind oder nicht in den tradierten Wissenschafts- und Publikationsbetrieb passen. In der Regel nimmt in vergleichbaren Veröffentlichungen die Beschreibung des Krankheitsbildes und die Diagnostik viel Raum ein. Hier werden jedoch neben einer mit einer gewissen Leichtigkeit vermittelten Darstellung differenzierter Pathologie, die auch dem diesbezüglich erfahrenen Leser beim Aktualisieren und Vertiefen dieser Themen Freude bereitet, salutogenetisch und ressourcenorientierte Betrachtungsweisen aufgezeigt. Besonders hervorzuheben ist jedoch, dass in einer Weiterführung in nahezu jedem Beitrag die Möglichkeiten verhaltenstherapeutischer Interventionen stimmig daraus abgeleitet und anschaulich dargestellt werden. Insgesamt kommt so etwas wie eine «positive weibliche Energie» - vielleicht auch «women power»zum Tragen: die Lektüre ist wohltuend, Kraft gebend, nicht nur auf die Pathologie, sondern auf Vorsorge und Therapie ausgerichtet.

Herausgekommen ist ein interessantes, vielseitiges, oft auch kurzweiliges, vor allem aber informationsträchtiges Buch, das - nicht nur Frauen - zum Weiterlesen anregt und immer wieder von scheinbar bewährten und eingespielten Denkweisen wegführt.

Diesem Buch ist zu wünschen, dass es als wesentliches Lehrbuch zu einem bislang wenig beachteten Thema seinen Platz in Forschung, Lehre und Therapieausbildung findet.

Eva Koppenhöfer/Dirk Lorenzen, Mannheim

\section{J.E. Young; J.S. Klosko; M.E. Weishaar}

\section{Schema Therapy - A Practitioner's Guide}

New York, Guilford, 2003, 436 S., USD 45,ISBN 1-57230-838-9

Young, Klosko und Weishaar beschreiben in Schema Therapy einen neuen, umfassenden Ansatz in der Psychotherapie. Dieser Ansatz eignet sich auch und besonders für Patienten mit tief greifenden Störungen und für die Bewältigung schwieriger Therapiesituationen.

Das Buch führt zuerst in die konzeptuellen Säulen der Therapie ein und beschreibt detailliert und anhand vieler eindrücklicher Beispiele die Struktur und Vielfalt schematherapeutischer Interventionen. Zwei abschließende Kapitel beschreiben Spezialgebiete der Schematherapie, nämlich die Konzeption und Behandlung von Borderline- und narzisstischen Persönlichkeitsstörungen.

Als Säulen der Therapie gelten die «früh erworbenen und im Erwachsenenalter hinderlichen Schemata» (early maladaptive schemas, EMS), Schemacoping, Schemamodi, die therapeutische Beziehung, besonders in der Form der «begrenzten elterlichen Fürsorge», und die gleichgewichtige Verwendung behavioraler, körperlich-emotionaler und kognitiver Methoden in Diagnostik und Therapie.

Anders als bei deutschsprachigen Autoren wurde der Ansatz aus der Analyse von Therapiebarrieren, -misserfolgen und -abbrüchen entwickelt: Die EMS wurden eingeführt, um Patienten gerecht werden zu können, deren Leid lange Zeit in ihrem Leben bestand und denen bloße Symptomlinderung weder Glück noch Zufriedenheit ermöglichten. Dabei ist ein Schema ein thematisch an einer frühen Entbehrung orientiertes Ganzes aus Körperreaktion, Gefühl, Kognition und Verhalten, welches durch Bedingungen ausgelöst wird, die Aspekte der traumatisierenden Ursprungssituation enthalten.

Die bisher gefundenen und im Buch explizierten 18 Schemata werden als besonders früh entstandene unkonditionale (z.B. emotionale Deprivation) oder als später entstandene konditionale Schemata (z.B. Unterwerfung) dargestellt, die es durch besondere Aktivität oder Anstrengung ermöglichen, einen Ausgleich bei sich oder der Umwelt zu erzielen.

Ein Schema kann ohne die Kenntnis des dazugehörenden Schemacopingstils und der konkreten Schemacopingreaktionen weder ein Störungsbild erklären, noch einen Behandlungsplan ermöglichen. Drei Schemacopingstile, Abfinden mit sowie Aushalten des Schemas, Schemavermeidung und Schemakompensation werden vorgestellt. Die Wechselbeziehungen zwischen Schemata und Schemacoping können dazu führen, dass etwa bei Schemavermeidung oder -kompensation ein Schema nicht ohne Weiteres erkannt wird. Da die Kenntnis und Bearbeitung relevanter Schemata für eine erfolgreiche Therapie unverzichtbar sind, werden Wege zur Erfassung schwer zugänglicher Schemata beschrieben.

Je tief greifender die Beeinträchtigungen eines Patienten sind, um so wahrscheinlicher ist es, dass unkonditionale, früh ent- 
standene Schemata eine Rolle spielen und eine große Zahl hinderlicher Schemata vorhanden ist. Die resultierende Wechselhaftigkeit der Patienten wird in der Schematherapie durch die Arbeit mit Schemamodi beantwortet. Schemamodi sind abgrenzbare Gesamtzustände, die nacheinander auftreten können und z.B. bei Borderlinepatienten in schneller Folge und als nicht integrierte Anteile der Person das gesamte Erleben und Verhalten, insbesondere die interpersonale Kommunikation, bestimmen. Die Autoren beschreiben aus der Kindheit stammende Modi, dysfunktionale Copingmodi, am destruktiven elterlichen Verhalten orientierte Modi und den in der Therapie besonders geförderten Modus des «gesunden Erwachsenen» und weisen Wege zum Umgang mit verschiedenen Modi.

Bei Patienten, die in ihrem Leben besonders viel entbehren mussten, wird die therapeutische Beziehung als begrenzte elterliche Fürsorge als Voraussetzung für Erfolg angesehen.

Das Buch hebt auch die Rolle der Schemata, Copingstile und Modi der Therapeuten für das Gelingen einer Therapie hervor. Es ist allen zu empfehlen, die sich für die Behandlung des gesamten Spektrums Psychotherapiesuchender entschieden haben.

Heinrich Berbalk, Hamburg

\section{F. Petermann (Hrsg.)}

\section{Lehrbuch der Klinischen Kinderpsychologie und -psychotherapie \\ 5., korrigierte Auflage \\ Göttingen, Hogrefe, 2002, 634 S., EUR 49,95 \\ ISBN 3-8017-1651-1}

Die klinische Kinderpsychologie ist von ständigen Veränderungen und Neuerungen gekennzeichnet. Die 5., korrigierte Auflage des Lehrbuches der Klinischen Kinderpsychologie und -psychotherapie trägt diesem Umstand Rechnung und bietet sowohl denjenigen, die sich neu in dieser Fachdisziplin orientieren möchten, als auch denjenigen, die sich über den aktuellen Forschungsstand informieren möchten, eine wertvolle, breit gefächerte und durch den strukturierten Aufbau gut handhabbare Hilfestellung.

So finden sich 21 Beiträge von 23 renommierten Autoren, welche vier Hauptbereichen zugeordnet sind und denen ein Einführungsbeitrag des Herausgebers vorangestellt ist, in dem Grundbegriffe und Trends der klinischen Kinderpsychologie und Kinderpsychotherapie pointiert dargestellt werden.

Der erste Hauptbereich widmet sich den Grundlagen, welche störungsübergreifend Gültigkeit und Relevanz besitzen. Klassifikation und Epidemiologie psychischer Störungen werden ebenso thematisiert wie entwicklungspsychopathologische Zusammenhänge, diagnostische Verfahren und Aspekte der Prävention und Gesundheitsförderung im Kindes- und Jugendalter.
Der zweite Teil des Lehrbuches beschäftigt sich mit emotionalen Störungen und Verhaltensstörungen, sprich hyperkinetischen Störungen, Aggression, Angst- und Zwangsstörungen sowie Depression. Neben Symptomatik, Nosologie, Epidemiologie und Verlauf sowie Erklärungsansätzen gehen die Autoren vor allem auf die Möglichkeiten der Intervention ein, so dass der praktisch interessierte Leser konkrete Handlungsvorschläge erhält.

Der dritte Hauptteil widmet sich kognitiven Störungen und Entwicklungsstörungen. Praxisnah werden neue Ergebnisse und Interventionsverfahren vermittelt zu neurophysiologischen und neuropsychologischen Störungen, psychischen Störungen infolge von Intelligenzminderungen, Störungen der Ausscheidung, umschriebenen Entwicklungsstörungen, tief greifenden Entwicklungsstörungen, umschriebener LeseRechtschreib-Störung.

Im vierten Teil werden chronische Krankheiten, psychosomatische Störungen (wie Essstörungen) und Schmerz diskutiert. Der Blick der Autoren ist auch hier insbesondere auf die Belastungen bei den Familien und auf mögliche Interventionsstrategien gerichtet, da der Herausgeber, wie er im Vorwort beschreibt, im Dialog mit den Autoren aller Beiträge wiederholt gefragt hat, wie man unter Beachtung der neuen Forschungsergebnisse die klinische Praxis verbessern und Kindern und Familien damit konkret besser helfen kann.

Die Veranschaulichung durch Tabellen, Übersichten, Abbildungen und Kästen wird dem Anspruch eines Lehrbuches ebenso gerecht wie die sich an die jeweiligen Texte anschlieBenden Zusammenfassungen und Verständnisfragen, die den Leser zur selbständigen Wissensüberprüfung anleiten. Zudem findet man zusätzlich zu den allgemeinen Literaturangaben zu jedem Thema Hinweise zur vertiefenden Lektüre.

Fazit: Das Handbuch stellt für Studierende, Neueinsteiger und Spezialisten gleichermaßen ein zentrales Standardwerk dar. Die klinische Kinderpsychologie ist eine für viele Disziplinen immer wichtiger werdende Fachdisziplin. Der Kinderverhaltenstherapie kommt in diesem Kontext eine besonders hervorzuhebende Rolle zu, die im vorliegenden Lehrbuch immer durch Praxisbeispiele unterstrichen wird. Auf diesem Hintergrund kann das Lehrbuch allen Intervenierenden nachdrücklich zur Lektüre empfohlen werden.

Stefanie Roos, Dortmund

\section{B. Strau $\beta$ (Hrsg.) \\ Psychotherapie bei körperlichen Erkrankungen. \\ Jahrbuch der Medizinischen Psychologie Nr. 21 \\ Göttingen, Hogrefe, 2002, 345 S., EUR 34,95 \\ ISBN 3-8017-1449-7}

Es ist die erklärte Absicht des Herausgebers, die neueren Entwicklungen bei der psychotherapeutischen Behandlung spezieller Krankheitsbilder darzustellen, um durch die Illustra- 
tion des möglichen Nutzens dem negativen Trend entgegenzuwirken, der derzeit innerhalb der Medizin hinsichtlich psychologischer Ansätze zu verzeichnen ist. Die Buchbeiträge schlagen in sechs Kapiteln den Bogen von epidemiologischen Fragen über Methodenbeschreibungen hin zu spezifischen Anwendungsbeispielen.

An den Anfang stellt B. Strauß eine hilfreiche Systematisierung psychotherapeutischer Interventionsverfahren und Indikationsgebiete körperlicher Erkrankungen. Am Beispiel der Krebserkrankung wird die Wirksamkeit von Gruppentherapien verdeutlicht. Der Autor diskutiert auch das Problem, dass trotz Erfolg versprechender Behandlungsangebote die Übernahme in die Klinikroutine nicht immer ausreichend gelingt.

Die große Bedeutung des Problems wird im Beitrag von Härter und Bengel aufgezeigt. Sie ermittelten die Auftretenshäufigkeit behandlungsbedürftiger psychischer Störungen bei kardiologischen Patienten in stationärer Rehabilitation und berichten eine mit ca. $20 \%$ hohe Rate. Im Beitrag von Petermann und Warschburger kommt die Interventionstechnik der Patientenschulung zur Sprache. Die Grundzüge dieser Intervention werden anhand von Asthma- und Neurodermitis-Patientenschulungen illustriert. Verres et al. stellen ein minimal invasives Verfahren vor und illustrieren die Vorgehensweise anhand von zwei Fallvignetten.

Ein weiteres Kapitel widmet sich dem anspruchsvollen Feld psychotherapeutischer Interventionen bei somatoformen Störungen. Der erste Beitrag beschreibt die Interventionsmöglichkeiten der Körperpsychotherapie (Joraschky et al.) und der funktionellen Entspannung. Die verhaltensmedizinische Behandlung wird im Beitrag von Timmer und Rief beschrieben und mit Evaluationsergebnissen untermauert. Gaab at al. stellen ein kognitiv verhaltenstherapeutischer Kurzzeittherapieverfahren an den Beispielen der «neuen» funktionellen Störungen (Chronic Fatigue Syndrome, Irritable Bowel Syndrome) vor. Erläutert wird die jeweilige Symptomatik sowie gängige Entstehungsmodelle dieser Störungsbilder. Die Verschiebung inhaltlicher Gewichte im Informationsverarbeitungsprozess bei somatoformen Patienten wird von Scholz aufgegriffen. Seine empirisch orientierten Ausführungen liefern Hinweise für die therapeutische Arbeit an den verzerrenden kognitiven Strukturen.
Der vierte Themenschwerpunkt widmet sich Onkologiepatienten. Psychotherapeutische Verfahren können bei diesen wesentliche Begleitmaßnahmen der medizinischen Behandlung sein. Der Beitrag von Schröder und Wolff beschreibt, wie Patienten mit einer edukativen Kurzzeitintervention erfolgreich auf eine allogene Knochenmarktransplantation vorbereitet werden konnten. Die Unterstützung Krebskranker mit psychodynamisch-psychotherapeutischen Methoden wird von Faller aufgegriffen. Abwehr- und Copingprozesse bei Krebs werden kurz eingeführt und Behandlungsprinzipien abgeleitet. Ein Fallbeispiel illustriert das Vorgehen. Evaluationsergebnisse werden dargestellt.

Kapitel 5 umfasst spezifische Anwendungsfelder. Dargestellt werden Ansätze zur Psychotherapie in der Dermatologie (Gieler und Stangier) und Neurologie (Leplow und Paetow). Bei den Ansätzen zum chronisch-subjektiven Tinnitus (Jäger und Lambrecht) und einer HIV-Gruppentherapie (Hüsler et al.) werden neben dem therapeutischen Prinzip jeweils auch Ergebnisse eigener Studien berichtet. Der Beitrag von Hautzinger rundet mit einer Darstellung psychotherapeutischer Ansätze in der Geriatrie das Themenfeld ab.

Der Band verdeutlicht die vielfältigen Möglichkeiten, mit psychotherapeutischen Methoden Erfolg versprechend in das Krankheitsgeschehen bei rein organischen sowie bei organisch gefärbten Störungen eingreifen. Insofern ist die Absicht der Herausgeber erfüllt worden, diese im klinischen Alltag vernachlässigten Möglichkeiten psychotherapeutischer Arbeit aufzuzeigen. Die wissenschaftlich-evaluative Untermauerung trägt zur Gesamtbedeutung des Buches bei. Damit wird der verbreiteten Ansicht, psychotherapeutische Ansätze seien empirisch zu wenig gesichert, entgegengearbeitet. Für die Praktiker tut sich deswegen gelegentlich die Relevanzlücke auf. Man merkt den gesammelten Beiträgen auch an, dass sie nicht auf ein spezifisches Thema hin geschrieben wurden. Hier hätte man sich manchmal eine konzisere Darstellung gewünscht.

In Zeiten der Ressourcenknappheit im Gesundheitssystem ist es verdienstvoll, auf die Stärken und Möglichkeiten einer Behandlungsweise hinzuweisen, die im Vergleich zu den stärker technisch und biochemisch orientierten therapeutischen Ansätzen in der Medizin sonst Gefahr läuft, in Vergessenheit zu geraten.

Lutz Mussgay, Bad Kreuznach 